\title{
PERSPECTIVAS DE CAMBIO DE LA POLITICA EXTERIOR PARAGUAYA
}

\author{
José Félix Fernández Estigarribia
}

Según el autor el primer obstáculo para un cambio en la política exterior paraguaya fue eliminado con el derrocamiento de Stroessner. En este sentido se enuncian los rasgos que tuvo su acción en lo internacional, marcada fundamentalmente por la propia voluntad del presidente y por una cancillería aferrada a los esquemas de la Guerra Fría.

Partiendo de la base de la interrelación entre lo interno y lo externo, se describe lo que ocurrió en el país después del golpe. Con respecto a esto se manifiesta la desconfianza existente tanto en sectores nacionales como en la sociedad internacional por el proceso de transición, siendo un gran obstáculo para lograr la plena democracia. El autor expresa que una mejor comprensión de la política internacional y del mundo sobre lo que ocurre en el país ayudaría a ello.

La política internacional del nuevo gobierno podría definirse como conservadora democrática. Esta idea la ejemplifica el autor analizando las relaciones con Brasil, Argentina y Estados Unidos.

Finalmente, enfatizando que aún no ha existido un debate sobre lo internacional, se enumeran algunas tendencias de la política exterior y se concluye que un nuevo planteamiento pasa un proceso de modernización del país y por el consenso.

El tema propuesto exige en el Paraguay algunas reflexiones: En un sentido amplio, el cambio ya está hecho. El derrocamiento de Alfredo Stroessner, la gran sorpresa de la sociedad internacional en 1989, significa un cambio sustancial.

La política exterior paraguaya, aunque enmarcada en ciertas premisas ideológicas, era también mucho de la voluntad del dictador, su peculiar visión del mundo, sus bajos intereses y aún sus humores. Si en toda política internacional hay un poco de las condiciones humanas de sus autores, en el Paraguay dada la gran concentración de poder personal que fue acumulando a lo largo de treinta y cuatro años, esto adquirió ribetes increíbles.

La última etapa de la administración depuesta estuvo plagada de acciones y episodios que lo prueban. Stroessner, en la necesidad psicológica que lo embargaba de convertirse en presidente vitalicio e instaurar su dinastía, fue perdiendo incluso el apoyo de antiguos 
José Félix Fernández Estigarribia / Perspectivas de cambio de la política...

sostenedores, para concluir absolutamente identificado con políticas como las de Taiwán, el racismo sudafricano y la dictadura de Corea del Sur a los cuales desaprobaba su proceso de transición. Hacia Chile existieron críticas por las debilidades de Pinochet al consentir el plebiscito.

Basta con recordar sus enfrentamientos con la administración Reagan, que jamás tuvo la intención de derrocarlo, y sólo pretendía aconsejarlo en temas como el tráfico de drogas, la corrupción generalizada y la necesidad de institucionalizar una salida, dada su avanzada edad. Este tipo de recomendaciones eran verdaderas provocaciones en la óptica de la dictadura. Stroessner, como en su caso Somoza, sólo recordaba antiguos favores y consideraba a los norteamericanos como traidores que no sabían corresponder a su antiguo gesto de enviar tropas a República Dominicana en 1965.

En ningún momento el general advirtió que la mayoría de los funcionarios del Departamento de Estado que se ocupaban del caso paraguayo estaban en la escuela cuando se produjeron aquellos acontecimientos y que Dominicana para ellos era un episodio "casi tan antiguo" como la guerra con Cuba.

En otro plano, las críticas de la Conferencia Episcopal paraguaya a su gestión gubernamental, lo llevaron a enfrentamientos con el propio Vaticano. Su ridículo intento de manipular la visita papal al Paraguay en mayo de 1988 lo llevó a una espectacular derrota cuya publicidad recorrió el mundo.

Ni siquiera Brasil, país que Stroessner atendía preferentemente, era muy escuchado. Incidentes como la permanencia del cónsul paraguayo en Paranaguá acusado de graves delitos, a pesar de las sugerencias de Itamaraty, y la continuación del contrabando de electrónica a Brasil que ponía en peligro la ley de protección a la informática brasileña, son ejemplos de una concepción internacional difícil de explicar en términos razonables. Debemos admitir que Brasil dejaba pasar muchas de estas cosas atendiendo a otros beneficios que para su política internacional le acarreaba la presencia de Alfredo Stroessner en la presidencia del Paraguay. Para muchos paraguayos es la última etapa de un episodio aún no concluido. ${ }^{1}$

\footnotetext{
${ }^{1}$ Para una mejor comprensión de ello sería importante la lectura de $A$ herencia de Stroessner, Brasil-Paraguay 1955-1980 de Alfredo Da Mota Menezes, Editorial Papirus, 1987. Aunque sin compartir todas sus conclusiones es un importante estudio de las relaciones paraguayo-brasileñas y del modo como Stroessner concebía esa política.
} 
La cancillería stronista seguía aferrada a esquemas de la Guerra Fría sin percibir los cambios que ocurrían en el mundo. Incluso, sostenía, había que resistir y presentar como peligro a todo el proceso de democratización del Cono Sur. Esta peculiar visión de lo que ocurría en los países limítrofes, en América Latina y en todo el resto del mundo, fue compartida por mucho tiempo por grandes sectores de la dirigencia paraguaya. Sin ser el único, Paraguay fue uno de los casos que pretendió explicar al resto del mundo cómo debían hacerse las cosas. Más adelante procuraremos indicar cuánto pesan estas rémoras del pasado para el diseño de una nueva política exterior.

Cada vez más solo, abandonado por sus aliados internacionales de otras épocas, molesto para muchos sectores de la sociedad internacional, el 10 de diciembre de 1988 el régimen paraguayo sufrió un traspié decisivo. En el día de los Derechos Humanos, y a pesar de haber apresado a la mayoría de la dirigencia opositora, las fuerzas contestatarias paraguayas ganaron la calle, y la policía no pudo derrotarlas. ¿Para qué servía un dictador, corrupto e incapaz, si ni siquiera era apto para controlar las manifestaciones?

Las Fuerzas Armadas ya no podían continuar con el desgaste que les ocasionaba seguir sosteniendo a ese figurón del pasado, que sólo ofrecía un permanente mirar para atrás.

Incluso sectores militares y marinos han aceptado públicamente que el régimen cayó por sus innumerables desaciertos, y las Fuerzas Armadas sólo dieron el empujón final.

Con su caída, queda abierta la posibilidad a la sociedad paraguaya de intentar un cambio en la política exterior. El obstáculo principal ha sido eliminado y ese fue el sentido de nuestra primera afirmación.

\section{La situación de Paraguay después del golpe del 2 de febrero}

No compartimos la tesis que la política exterior es un reflujo de su política interna. La interacción es recíproca, y mucho de la política interior es consecuencia de fuerzas de la sociedad internacional que operan sobre ella.

Es imposible a esta altura tener una perspectiva adecuada de todo lo que está sucediendo. "Quien quiera que aspire a aprehender directa e inmediatamente las realidades de la vida política deberá 
José Félix Fernández Estigarribia / Perspectivas de cambio de la política...

contentarse con una visión aproximada de las cosas y tendrá que dejar un lugar irreductible a la interpretación personal".

Aceptando esta premisa es importante describir a grandes rasgos lo que sucedió en el Paraguay desde el golpe.

La misma desconfianza de la sociedad internacional hacia el nuevo gobierno paraguayo, existió y aún subsiste en sectores de la vida política y social del Paraguay.

Muchos mencionaban el origen militar del nuevo presidente, sus vínculos de parentesco con el tirano derrocado y el haber sido uno de los principales sostenes de su régimen durante muchísimos años. Nadie o casi nadie trajo a colación que este mismo fenómeno existió en casi todos los procesos de derrocamiento de dictadores, muchos de ellos depuestos por figuras militares de su entorno. Perón en su segunda presidencia, Marcos en Filipinas podrían ser muestras tomadas al azar.

El grupo militar triunfante se apoyaba en el sector civil del Partido Colorado, conocido como "tradicionalistas", el único sector colorado en ser apartado por Alfredo Stroessner. Este grupo había sido desplazado en la Convención de Agosto de 1987, y muchas de sus figuras habían continuado de un modo u otro en la estructura gubernamental que acababa de ser derrocada.

La proclama de las Fuerzas Armadas, explicatorias del golpe, si bien introducían valiosos puntos, como la defensa de los derechos humanos, la necesidad de democratizar al país, el cese de la persecución a la Iglesia católica, volvía a introducir el tema de la reunificación del Partido Colorado.

Para numerosos observadores de Paraguay, significaba la continuación de un lamentable modelo político que institucionalizaba la imbricación de un partido político con el ejército, unido a la afiliación obligatoria de los integrantes de las Fuerzas Armadas en ese mismo partido.

$\mathrm{Ni}$ en lo nacional y menos en lo internacional ello puede ser presentado como un modelo democrático.

La decisión de llamar a elecciones dentro de los tres meses como lo preceptuaba la Constitución no ayudaba a disipar los temores que se intentase repetir el modelo depuesto con otros actores.

El debate se produjo en todos los niveles. Tal vez el más impactante estuvo dentro del Partido Liberal Radical Auténtico, la

\footnotetext{
${ }^{2}$ Marcel Merle, Sociologia de las Relaciones Internacionales, (Alianza Universidad, 1984), p. 28.
} 
mayor fuerza opositora. Un fuerte sector del mismo impugnaba la participación en las elecciones. La decisión del partido a concurrir fue tomada en una convención transmitida por televisión en todo el país, y en esta interna nadie dejó de tomar posición. Es posible que la decisión del partido de concurrir a comicios estuvo muy influida por su presidente y principal líder, Domingo Laíno, quien era partidario de la concurrencia. Sectores de intelectuales que rodean al mismo lo apoyaron e influyeron en la convención.

Se basaban en el reconocimiento del partido, proscrito por muchos años, de otras fuerzas políticas, la restauración de las libertades públicas, y la reapertura de los medios de comunicación clausurados. ${ }^{3}$

La Iglesia católica, aunque no se pronunció sobre el punto comenzó un evidente acercamiento al nuevo gobierno, lo que parecía alentar a aceptar las reglas del juego.

Sobre las fuerzas opositoras existió una evidente presión de diversos actores de la política internacional, para intentar un nuevo camino aceptando las imperfecciones del sistema.

La Internacional Socialista y la Democracia Cristiana no estuvieron ajenas a ello y organizaron misiones al Paraguay en apoyo de la tesis. Por su parte los gobiernos de Argentina, Brasil y Uruguay, no ocultaban su interés en el proceso, lo que calificaban a través de medios oficiosos de la salida posible. Tomarle la palabra a Rodríguez pareció ser la consigna.

Esta política continuaría después de las elecciones, con la presencia en Asunción de los presidentes Sarney, Alfonsín y Sanguinetti, el día de la asunción oficial al mando. ${ }^{4}$

Las elecciones que consagraron el triunfo de Rodríguez fueron definidas por él mismo "como libres pero no limpias". A partir de ese momento los paraguayos iniciaban otra vez una apuesta con su destino. Uno de los principales obstáculos reside en la poca fe que en el exterior se tiene sobre ellos para desenvolverse en democracia. Incluso sectores progresistas los califican "a priori" como destinados a manejarse con dictaduras. Una mala lectura de su historia, una falta absoluta de análisis de cómo los factores internacionales operaron

\footnotetext{
${ }^{3}$ Laíno acaba de reconocer a la prensa que mucho influyó en su decisión la opinión de Carlos Andrés Pérez, con quien se entrevistara unos días después del golpe. $A B C$-COLOR, 26 de septiembre de 1989.

${ }^{4} \mathrm{El}$ presidente Sanguinetti ha continuado con esa política y ha viajado con el presidente Rodríguez a Bolivia para la transmisión del mando a Paz Zamora. Numerosos sectores ven con simpatía la buena influencia del mandatario uruguayo.
} 
José Félix Fernández Estigarribia / Perspectivas de cambio de la política...

en su contra, alentando a las fuerzas reaccionarias de su interior, parece haberlos descalificado.

Frente a ello, en el Paraguay de hoy existe un razonable optimismo de que esta vez se pueda lograr lo que anhela una inmensa mayoría del país, llegar a la democracia. Una mejor comprensión de la política internacional, ayudaría a ello, y a la inversa una mejor comprensión de la sociedad internacional de lo que ocurre dentro de sus fronteras sería trascendente.

\section{La preocupación por lo internacional}

El nuevo gobierno, manteniendo una política, conservadora, ha comenzado a preocuparse de lo internacional. Ha percibido la mala imagen de la dictadura anterior y procura superarla.

En su seno se debaten dos tendencias. La primera, dominada por los políticos del gobierno, con su epicentro en dos figuras: el canciller Luis María Argaña, hombre del tradicionalismo y el embajador Miguel Angel González Casabianca, adscrito a la presidencia de la República. Por curiosidad, y aunque lo niegan, ambos son presumibles candidatos a la presidencia de la República.

Es posible que les preocupe los pocos vínculos de su partido con el mundo internacional. González Casabianca, que vivió 25 años en el exilio, conoce esta problemática y tiene conciencia de ello. Ninguno ignora que en esto los liberales radicales auténticos y los febreristas le llevan ventaja.

Con respecto a la segunda tendencia, el equipo que maneja el ministro Zuccolillo, responsable del Ministerio de Industria y Comercio, ha procurado atraer capitales al país, presentando un modelo privatizador y modificando la Ley de Inversiones Extranjeras.

Ambos grupos carecen, por lo menos, aparentemente, de un marco teórico y si el mismo existe podría ser definido como conservador democrático. En el análisis de algunos tópicos procuraremos desarrollar esta afirmación.

\section{La relación con Brasilia, Washington y Buenos Aires}

Paraguay, por su condición geográfica, ha sido poderosamente influido desde Brasil y Argentina. Alguien definió a su historia como la avanzada contención del imperio español al portugués. Como todo simplismo debe ser desechado, mas no olvidado. 


\section{Brasil}

Aunque no se lo diga, nadie olvida en Paraguay los vínculos y los apoyos de diversos gobiernos brasileños, incluso democráticos, al régimen autoritario de Alfredo Stroessner. Brasil está en deuda con la democracia paraguaya, y muchos creen que esto sólo cambiaría con las elecciones directas en Brasil. Los demócratas paraguayos apuestan a la democracia brasileña. A eso se añade el asilo del gobierno brasileño a Stroessner. Incluso en el poderoso vecino vive el coronel Gustavo Stroessner, hijo del dictador reclamado por la justicia paraguaya. Ninguno de estos elementos ayudan a una relación muy fluida. Los diplomáticos brasileños se escudan en que el asilo fue pedido por el propio gobierno paraguayo, y el episodio tardará cierto tiempo en ser esclarecido.

Aun así, es evidente que el gobierno paraguayo $-\mathrm{y}$ en esto encuentra consenso en la opinión pública- está decidido a no convertirse en aliado incondicional de Brasil como lo diseñaron algunos teóricos brasileños y lo aprobaron Stroessner y su canciller de entonces, Raúl Sapena Pastor.

El propio ministro de Relaciones Exteriores lo señala cuando dice: "Hay, por ejemplo, entre los países vecinos una política de equilibrio, porque en un momento dado, nosotros prácticamente veíamos que nuestro destino estaba enmarcado hacia un rumbo, hacia un sólo país, convirtiéndonos en cierto momento en un satélite de la política internacional de ese país. Hoy día, nosotros estamos restaurando el equilibrio histórico de manera que Paraguay no tenga sino como identidad, como nación libre y soberana, relaciones con todos los países del mundo, sin privilegios de ninguna clase". 5

La alusión a Brasil es obvia. Mas, esto no es una expresión de deseos. Brasil en su relación con Paraguay tiene los problemas propios de la herencia de Stroessner. Itamaraty fue increíblemente ingenuo al creer que lo de Stroessner duraría eternamente y a los diplomáticos brasileños les cuesta la nueva relación. Existen puntos concretos que tendrán que ser redefinidos.

Lo inminente es la renegociación de la deuda externa, cuya discusión comenzara ya en época de Stroessner. El acuerdo, firmado por el actual gobierno, como corolario de la discusión anterior, tiene

\footnotetext{
${ }^{5}$ Declaración del canciller Luis María Argaña. $A B C-C O L O R, 27$ de septiembre de 1989, p. 11.
} 
José Félix Fernández Estigarribia / Perspectivas de cambio de la política...

aspectos positivos, como sería la posibilidad de comprar los títulos de la deuda externa brasileña por el Paraguay y canjearlos -como lo prevee el contrato- por títulos de la deuda brasileña.

Sin embargo, el contrato establece los intereses a la tasa Libor, muy superior a la establecida en los anteriores préstamos y por sobre todo en las negociaciones no se ha tenido en cuenta para nada la ilegalidad de gran parte de esas obligaciones, producto -mucha de ella- de escandalosos negociados que hoy se discuten en la justicia paraguaya y al que no estuvieron ajenos los acreedores en ciertos casos. ${ }^{6}$ Este acuerdo está a la consideración del nuevo Parlamento paraguayo.

A más largo plazo, en esferas políticas y económicas del Paraguay, se habla de la necesidad de renegociar las cláusulas del Tratado de Itaipú, con el cual se construyera la fabulosa represa. La mayoría de los paraguayos creen que dicho instrumento es desfavorable a los intereses nacionales. Es posible que todo esto, unido a la presencia de miles de brasileños dentro de las fronteras, en tierras muchas de ellas reclamadas por campesinos paraguayos, que no las poseen, da origen a reclamos y conflictos que de ser solucionados con amplio espíritu integracionista se agrandarían innecesariamente.

\section{Argentina}

Para el actual gobierno, y para la oposición paraguaya, el presidente Alfonsín representó la solidaridad democrática latinoamericana y un apoyo en la lucha contra el régimen. Ni él ni su canciller Caputo visitaron Asunción mientras estuvo el dictador.

El gobierno paraguayo no puede olvidar el gesto de Alfonsín al haber sido el primer gobierno que lo reconoció. Desde el primer momento Caputo no ocultó su apuesta a consolidar el proyecto que se abría. Viajó a Asunción, y nuevamente acompañó al presidente Alfonsín en el día de la asunción al mando después de las elecciones. En privado, los paraguayos, oficialistas y opositores de hoy, ponderan su gestión.

\footnotetext{
${ }^{6}$ Debe señalarse que el presidente Rođríguez en su alocución a la Asamblea General a las Naciones Uniđas lo presentó de manera diferente: "Es así como, recientemente, hemos renegociado una parte importante de nuestra deuda externa con otro país latinoamericano, la República Federativa del Brasil. El ajuste de esta deuda podría ser considerado como un ejemplo de que siempre existen soluciones cuando se da la voluntad política de cooperación". $A B C-C O L O R, 27$ de septiembre de 1989.
} 
El triunfo de Menem, cuyos vínculos con el presidente Stroessner eran públicos y notorios, pudo afectar esta nueva luna de miel. La pasmosa habilidad de Menem para desdecirse parece haber satisfecho al gobierno paraguayo, empeñado a su vez en que nada perturbe la nueva buena relación. Incluso el nombramiento del embajador Floro Bogado en Asunción fue absorbido por el gobierno, que lo dejó pasar tolerantemente, a pesar de la prensa adversa con que fue recibido el nuevo titular de la misión diplomática argentina. ${ }^{7}$

Argentina no está en condiciones de aportar ayuda sustancial al gobierno paraguayo, pero un frente de fricción ha sido superado, y más bien todo indica que las relaciones serían fluidas y crecientes.

\section{Estados Unidos}

El gobierno de Estados Unidos de América tardó en reconocer el gobierno paraguayo. Antiguas acusaciones contra el nuevo presidente de vínculos contra el narcotráfico habrían influido en ello. A ello debemos añadir que el golpe se produjo cuando la administración Bush intentaba recomponer sus vínculos con el stronismo.

La firme actitud del presidente Rodríguez de combatir al narcotráfico parece haber convencido a los latinoamericanos. El nuevo gobierno parece no transar en este punto. Lo ha reiterado recientemente en las Naciones Unidas: "En mi país, que tampoco se ve libre de esta amenaza, mi gobierno procedió inmediatamente que se tuvo noticia de plantaciones clandestinas de marihuana ubicadas en el territorio paraguayo, a la destrucción de las zonas de cultivo con aviones fumigadores, contando para el efecto, con la cooperación del gobierno de los Estados Unidos de América en una campaña enérgica para erradicar cualquier manifestación del tráfico de estupefacientes. Mi gobierno no permitirá que el suelo paraguayo sea utilizado como vía para el tránsito de las drogas y de los narcotraficantes, y dentro de sus limitaciones económicas, dispondrá de todos los medios para prevenir y erradicar esta situación"."

El gobierno paraguayo ha pedido ayuda al norteamericano para la reforma agraria, uno de los puntos candentes de la realidad para-

\footnotetext{
${ }^{7}$ Floro Bogado durante su gestión como embajador đe Formosa, realizó diversas manifestaciones de apoyo al presidente Stroessner. Asistió a actos del Partido Colorado con el pañuelo rojo al cuello, en momentos en que éste estaba totalmente dominado por el grupo stronista.

8Discurso del presidente Rodríguez en el $44^{\circ}$ Período Ordinario de la Asamblea General de las Naciones Unidas.
} 
José Félix Fernández Estigarribia / Perspectivas de cambio de la política...

guaya de hoy. Sin embargo, la ayuda no se ha materializado y no se han firmado nuevos convenios de ayuda ni cooperación.

El gobierno norteamericano parecía apoyar el proceso de la democracia. En ocasión del arribo del comandante de la Flota de los Estados Unidos de Ámérica en el Atlántico Sur, contraalmirante John R. Dalrymp a Asunción, el embajador de los norteamericanos expresó: "Está llegando a un nuevo Paraguay. En un Paraguay que va camino a la democracia. Un nuevo Paraguay en donde las Fuerzas Armadas juegan un papel institucional en defensa de la patria y no un papel político".

A pocos días de un pronunciamiento militar que advierte la preocupación de las Fuerzas Armadas por la marcha del proceso y se refiere especialmente al Partido Colorado, cuya crisis preocupa a todos, las palabras del embajador parecen ser la expresión de lo que el gobierno norteamericano desearía de que fuesen, esto sin descartar que por primera vez en muchos años en las Fuerzas Armadas del Paraguay hay un calificado grupo de militares que no desean verse envueltos en las alternativas políticas del partido de gobierno, lo que significa el comienzo de un proceso de institucionalización. Lógicamente esto tendrá impacto en lo internacional.

\section{Las tendencias de la política exterior}

La cancillería paraguaya ha tenido poco tiempo aún para juzgarla definitivamente. Enfrascada en numerosos problemas internos es difícil percibir exactamente cuál es su política.

Aun así podemos adelantar algunos juicios. Los mismos podrían ser desmentidos por acontecimientos nuevos, como ha sucedido en otras facetas de la nueva política paraguaya.

Se percibe como muy lento el proceso hacia la integración latinoamericana, esto obedecería a un análisis de relaciones internacionales demasiado clásico.

Al percibir exageradamente el rol de los Estados, y concebir a estos en permanente lucha por el poder, temas como el de la integración latinoamericana son mirados con desconfianza.

No se han dado pasos significativos en materia de acercamiento . al proceso de integración de Argentina, Brasil y Uruguay. Lo curioso

9Palabras del embajador de los Estados Unidos de América Timothy Towel.ABC-COLOR, 2 de octubre de 1989, p. 10. 
de esa actitud es que el país sufre los embates del contrabando, lo que convierte en un país abierto y poco debería temer de esta integración. Los industriales paraguayos más progresistas así lo han expresado.

No se han registrado cambios en las relaciones con nuevos países y no se vislumbra que se establezcan lazos con países socialistas con los cuales no se tienen ni relaciones diplomáticas. Es posible que Cuba y Nicaragua sean considerados como enemigos.

El gobierno ha reducido su relación con Panamá al nivel consular, siguiendo en esto la línea de otros países americanos.

Más preocupación causa que no hay signos de acercarnos al Grupo de los Ocho, lo que podría hacerse sin integrarlo, compartiendo su política en relación con Centroamérica. Lo mismo podría decirse respecto al Grupo de Cartagena.

Es obvio que en estas condiciones aún no se ha registrado un debate nacional sobre lo internacional. Mónica Hirst escribía cuando comenzaba la transición brasileña: "La aparición de estas cuestiones en una coyuntura de revitalización política representa en sí mismo un nuevo potencial de acción y de pensamiento tanto del Estado como de la sociedad civil". ${ }^{10}$ Es posible que todo esto pueda darse solamente dentro de la evolución de una sociedad paraguaya que busca modernizarse. En Paraguay esto pasa por arribar a un consenso dentro de la política paraguaya que permita llegar a elecciones que definan su futuro en lo nacional e internacional. Una vez más, los paraguayos luchan por la utopía, "mas es deber de todo científico luchar por ella".

\footnotetext{
10 Mónica Hirst, Pesos y medidas de la politica exterior brasileña. AmÉrica Latina. Polfticas exteriores comparadas, (Grupo Editor Latinoamericano).
} 